\title{
MEDICAL PROCEDURE BONE MARROW ASPIRATION AND BIOPSY
}

\author{
A.I. Onaga \\ Department of Medicine, University College Hospital, Ibadan
}

\section{Introduction}

The bone marrow examination is an essential investigation for the diagnosis and management of many disorders of the blood and bone marrow. The aspirate and trephine biopsy specimens are complementary and when both are obtained, they provide a comprehensive evaluation of the bone marrow. The final interpretation requires the integration of peripheral blood, bone marrow aspirate and trephine biopsy findings, together with the results of supplementary tests such as, immunophenotyping, cytogenetic analysis and molecular genetic studies as appropriate, in the context of clinical and other diagnostic findings. It is recommended that both BM aspirate and biopsy be routinely performed so that respective findings can be correlated. However, in some clinical situations, a BM aspirate alone may suffice.

\section{History of bone marrow examination}

The first bone marrow biopsy was reported in 1905 by Pianese. It was performed in an infant with leishmaniasis. In 1909, Pianese trephined the tibia and femur, aspirating the marrow with a needle attached to a syringe. In 1923, Seyfarth used a surgical trephine to obtain marrow from the ribs and the sternum. Because trephination resulted in excessive bleeding and infection, it was not a generally well-accepted procedure. Airinkin, in 1927, eliminated the trephine complications by using a short lumbar needle to puncture the manubrium and sternum. He obtained a marrow aspiration by placing a syringe on the needle hub. In 1945, Vandenberghe and Blitstein were the first to use the iliac crest to obtain bone marrow and Heidenreich obtained marrow from the spinous processes. In 1952, Bierman used the posterior iliac crest as the site for bone marrow aspiration, claiming it to be a safe site.

\section{Indications for bone marrow examination}

1. Investigation of unexplained anaemia, abnormal red cell indices, cytopenias or cytoses.

2. Investigation of abnormal peripheral blood smear morphology, suggestive of bone marrow pathology.

3. Diagnosis, staging and follow-up of malignant haematological disorders (e.g. acute and chronic leukaemias, myelodysplastic syndromes, chronic myeloproliferative disorders, lymphomas, plasma cell myeloma, amyloidosis, mastocytosis).

4. Investigation of suspected bone marrow metastases.
5. Unexplained focal bony lesions on radiological imaging.

6. Unexplained organomegaly or presence of mass lesions inaccessible for biopsy.

7. Microbiological culture for investigations of pyrexia of unknown origin or specific infections, e.g. miliary tuberculosis, leishmaniasis, malaria.

8. Evaluation of iron stores.

9. Investigation of lipid/glycogen storage disorders.

10. Exclusion of haematological disease in potential allogeneic stem cell transplant donors.

\section{Contraindication}

Hemophilia and other related disorders.

\section{Preparations prior to the procedure}

1. The procedure should be explained in detail to the patient. The past clinical history of the patient should be obtained, any allergies and co-morbidities documented and any premedications explained.

2. Informed consent should be obtained from the patient. Consent should also be obtained, according to local guidelines, for the use of any specimens for non-diagnostic purposes, e.g. research, tissue banking, education or quality assurance.

3. A blood count and smear should be obtained if these have not been collected in the previous 2 days.

4. Adequate sedation and analgesia determined

5. Assessment of thrombocytopenia or coagulopathic risks, which may require platelet transfusion support or the reversal of anticoagulation.

6. Consider site for BM examination carefully.

\section{Choosing a site}

The preferred anatomic site for BM aspiration and trephine biopsy is the posterior iliac crest. The anterior iliac crest can be used if the patient is immobile. The medial surface of the tibia can also be used in infants. A sternal aspirate may be appropriate in certain circumstances, e.g. if the patient is immobile, has received radiotherapy to the pelvis or other sites have yielded a 'dry tap' or if a trephine biopsy is not required. Sternal aspiration should only be performed by an experienced operator who is aware of the risk of cardiac tamponade. Sternal aspiration should not be attempted in patients with suspected plasma cell myeloma or other disorders associated with bone re- 
sorption. If there is a known focal bone lesion (from radiological imaging), diagnostic information may be obtained if a needle aspirate and bone biopsy is also performed at the site.

\section{The procedure}

It is recommended that the aspirate and trephine biopsy be obtained using the respective needles separately, and not through a trephine needle. If the aspirate is performed with a trephine needle, the aspirate sample may be haemodiluted. If the biopsy is then performed using the same needle, the trephine core may be damaged or haemorrhagic.

\section{The procedure - Bone marrow aspiration}

1. Special attention to positioning of the patient should be given to immobile patients, obese patients, paediatric patients or infants, patients with lytic bone lesions or BM necrosis, or those who have had prior radiotherapy. The prior preparations should be ensured.

2. The second and third fingers on the hand not being used to insert the needle should be placed on the iliac crest or spine and the needle inserted between them. The needle and stylet are pushed into the bone with a slight rotary motion. When it is felt that the needle is firmly in place, the stylet is removed and a 10 - or $20-\mathrm{ml}$ plastic syringe is attached. The patient is then told that he/she may feel an unpleasant sensation, and the plunger of the syringe is pulled back vigorously with no more than $0.5 \mathrm{ml}$ of bone marrow and blood aspirated

3. The 10- or 20-ml plastic syringe, attached to the aspiration needle provides adequate negative pressure.

4. Bone marrow smears should be prepared immediately following aspiration and a report given.

\section{The procedure - Bone marrow biopsy}

The BM trephine biopsy may be performed either before or after the aspirate. The length of the core from an adult should be at least $2 \mathrm{~cm}$.

A small incision with a scalpel blade is made before insertion of the biopsy needle and stylet. A different site should be chosen for the biopsy, if it is performed after the aspiration. After insertion of the biopsy needle into the bone, the stylet is removed. The needle is then advanced between 1 and $2 \mathrm{~cm}$, depending on the size of the patient. This is performed with very little rotation of the needle. After advancing the needle, the hub is rotated a few times in one direction, followed by a few times in the other direction. The needle is inserted a small additional amount to break the attachment, and the rotations are repeated. The thumb is then placed on the hub of the needle, and the needle is extracted with slight lateral movements accompanying the removal. The bone marrow biopsy is then placed on a slide, where imprints are made before processing for cytologic investigations.

After decalcification, the biopsy specimen is embedded in paraffin wax and sections cut on a microtome. The recommended thickness of sections is two to three microns. At least six sections should be cut at three levels: $25 \%, 50 \%$, and $75 \%$ into the cross-sectional diameter of the core, and serial sections mounted stepwise on glass slides. Additional sections need to be cut if IHC or histochemical stains are required.

\section{Complications}

Rarely mortality may follow this procedure. A needle may break; bleeding may follow especially in thrombocytopenic patients. Infections may be introduced and rarely pulmonary thromboembolism may complicate the procedure.

\section{Interpretation of results}

A hematology consult is very useful in interpreting results where the clinical picture and all blood and bone marrow results are reviewed to reach a diagnosis.

\section{The recommended bone aspirate report}

Name of institution.

Unique specimen identifier (laboratory accession number).

Details of patient: surname, first name(s), identification number, age or date of birth, gender, contact details (e.g. address, hospital location).

Name of responsible physician.

Name of requesting doctor.

Date of procedure.

Significant clinical history including physical findings, recent chemo/radiotherapy, cytokine therapy and pertinent lab results.

Indication for bone marrow examination.

Procedure performed (aspirate/trephine biopsy).

Anatomic site of aspirate/biopsy.

Ease/difficulty of aspiration.

Blood count: Haemoglobin concentration, total and differential white cell count (neutrophils, eosinophils, basophils, monocytes, lymphocytes) and platelet count. Blood smear description and diagnostic conclusion.

Cellularity of particles and cell trails.

Nucleated differential cell count.

Total number of cells counted.

Myeloid:erythroid ratio.

Erythropoiesis.

Myelopoiesis.

Megakaryocytes.

Lymphocytes. 
Plasma cells.

Other haemopoietic cells.

Abnormal cells (e.g. blast cells, metastatic infiltrates).

Iron stain.

Cytochemistry.

Other investigations (e.g. cytogenetics, PCR, FISH, microbiology).

Summary of flow cytometry findings, if available.

Conclusion.

WHO classification (if relevant).

Disease code.

Signature and date of report.

The recommended bone marrow trephine report Name of institution.

Unique specimen identifier (laboratory accession number).

Details of patient: surname, first name(s), identification number, age or date of birth, gender, contact details (e.g. address, hospital location).

Name of responsible physician.

Name of requesting doctor.

Date of procedure.

Significant clinical history including physical findings, recent chemo/radiotherapy, cytokine therapy and pertinent lab results.

Indication for bone marrow examination.
Procedure performed (aspirate/trephine biopsy).

Anatomic site of aspirate/trephine biopsy.

Aggregate length of biopsy core.

Adequacy and macroscopic appearance of core.

Percentage and pattern of cellularity.

Bone architecture.

Location, number, morphology and pattern of differentiation for erythroid, myeloid, megakaryocytic lineages, lymphoid cells, plasma cells and macrophages. Abnormal cells and/or infiltrates.

Reticulin stain.

Immunohistochemistry.

Histochemistry.

Other investigations (e.g. FISH, PCR).

Conclusion.

Disease code.

Signature and date of report.

\section{REFERENCES}

1. S.H. Lee, W.N. Erber, A. Porwit, M. Tomonaga, L.C. Peterson

ICSH guidelines for the standardization of bone marrow specimens and reports. INTERNATIONAL JOURNAL OF LABORATORY HEMATOLOGY, 2008

2. Hoffman: Hematology: Basic Principles and Prac tice, 3rd ed., 2000 Churchill Livingstone, Inc.

\section{ANSWERS}

\section{1. (A) CORRECT.}

Food allergies are typically a form of type I hypersensitivity reaction. The allergens react with $\operatorname{IgE}$ bound to mast cells, mainly in skin and gastrointestinal tract

\section{2. (C) CORRECT.}

These non-specific findings put together suggest possible autoimmune disease. An ANA is a good way to begin the workup, then more specific tests can be ordered. Antigen-antibody complexes of many autoimmune diseases can precipitate in a variety of locations, typically trapped in basement membranes in sites such as skin, synovium, pleura, pericardium, and glomeruli.

\section{3. (E) CORRECT.}

Recurrent bacterial infections suggest a lack of Bcell immune function with lack of gamma globu- lin production. Once maternal antibody is gone, the disease is manifested more severely.

\section{4. (D) CORRECT.}

He has hyper-IgM syndrome, an X-linked disorder. The CD40-CD40L interaction is involved with isotype switching from IgM production, thus accounting for his lack of $\operatorname{IgG}$ and $\operatorname{IgA}$. This accounts for bacterial infections. However, some degree of cell mediate immune defect is present as well.

\section{5. (D) CORRECT.}

Polymyositis-dermatomyositis is present. The violaceous skin rash is often quite subtle and limited to the face. 